\title{
Michigan Particle Swarm Optimization for Prototype Reduction in Classification Prob- lems
}

\author{
Alejandro CERVANTES and Inés GALVÁN and Pedro ISASI \\ Department of Computer Science \\ University Carlos III de Madrid \\ Avda. Universidad, 30 \\ 28911 Leganés, Madrid, Spain \\ $\{$ alejandro.cervantes, inesmaria.galvan,pedro.isasi $\}$ @uc3m.es
}

Received February 172009

\begin{abstract}
This paper presents a new approach to Particle Swarm Optimization, called Michigan Approach PSO (MPSO), and its application to continuous classification problems as a Nearest Prototype (NP) classifier. In Nearest Prototype classifiers, a collection of prototypes has to be found that accurately represents the input patterns. The classifier then assigns classes based on the nearest prototype in this collection. The MPSO algorithm is used to process training data to find those prototypes. In the MPSO algorithm each particle in a swarm represents a single prototype in the solution and it uses modified movement rules with particle competition and cooperation that ensure particle diversity. The proposed method is tested both with artificial problems and with real benchmark problems and compared with several algorithms of the same family. Results show that the particles are able to recognize clusters, find decision boundaries and reach stable situations that also retain adaptation potential. The MPSO algorithm is able to improve the accuracy of 1-NN classifiers, obtains results comparable to the best among other classifiers, and improves the accuracy reported in literature for one of the problems.
\end{abstract}

Keywords Nearest Neighbor Classification, Swarm Intelligence, Data Mining, Metaheuristics. 


\section{$\S 1 \quad$ Introduction}

The Particle Swarm Optimization algorithm ${ }^{1)}$ is a biologically-inspired algorithm motivated by a social analogy. The algorithm is based on a set of potential solutions which evolves to find the global optimum of a real-valued function (fitness function) defined in a given space (search space).

The PSO algorithm uses a population of particles which move in a multidimensional space that represents the space of solutions for the problem. Particles have memory, thus retain part of their previous state. There is no restriction for particles to share the same point in the search space, but in any case their individuality is preserved.

The basic PSO uses a real-valued multidimensional space as search space, and evolves the position of each particle in that space using (1) and (2).

$$
\begin{aligned}
& v_{i d}^{t+1}=\chi\left(w \cdot v_{i d}^{t}+c_{1} \cdot \psi_{1} \cdot\left(p_{i d}^{t}-x_{i d}^{t}\right)+c_{2} \cdot \psi_{2} \cdot\left(p_{\mathrm{gd}}^{t}-x_{i d}^{t}\right)\right) . \\
& x_{i d}^{t+1}=x_{i d}^{t}+v_{i d}^{t+1} .
\end{aligned}
$$

Where the meanings of symbols are:

$$
\begin{array}{ll}
v_{i d}^{t} & \begin{array}{l}
\text { component in dimension } d \text { of the } i^{t h} \text { particle } \\
\text { velocity in iteration } t
\end{array} \\
x_{i d}^{t} & \text { same for the particle position } \\
c_{1}, c_{2} & \text { constant weight factors } \\
p_{i} & \text { best position achieved so far by particle } i \\
p_{\mathrm{g}} & \text { best position found by the neighbors of particle } i \\
\psi_{1}, \psi_{2} & \text { Random factors in the }[0,1] \text { interval } \\
w & \text { inertia weight } \\
\chi & \text { constriction factor }
\end{array}
$$

The neighborhood of the particle may either be composed of the whole swarm ("gbest" topology) or only a subset of the swarm ("lbest" topologies). Also, some versions of PSO use dynamic neighborhoods, where the relationship between particles changes over time: Suganthan ${ }^{2)}$ proposed a swarm with a local neighborhood whose size was gradually increased; Brits ${ }^{3)}$ justifies the introduction of topological neighborhoods when searching for multiple solutions to multimodal problems using niching techniques. A dynamic neighborhood has been proposed by $\mathrm{Hu}$ and Eberhart ${ }^{4)}$ for multi-objective optimization.

There are also some good theoretical studies of PSO ${ }^{5,6,7,8)}$ which address the topics of convergence, parameter selection and trajectory analysis. 
The purpose of this paper is to use the continuous PSO for the selection of prototypes in nearest neighbor learning. Some work has already been done concerning PSO with classification problems. Sousa ${ }^{9)}$ uses PSO to extract induction rules to classify data; the standard PSO algorithm is run several times, extracting a single rule each time and using only unclassified patterns for the subsequent iterations. In previous work by the authors ${ }^{10,11)}$, induction rules are encoded using the binary version of PSO and both a single and an iterated version of the algorithm. Wang ${ }^{12,13)}$, has used a standard PSO for rule extraction in discrete classification problems. There is also some work in fuzzy rule extraction using PSO ${ }^{14)}$. Recently ${ }^{15)}$ PSO has been used to locate cluster centers for classification.

In this work we use Nearest Neighbor (NN) classification. NN is a lazy learning method where the class assigned to a pattern is the class of the nearest pattern known to the system, measured in terms of a distance defined on the feature (attribute) space.

In 1-NN with Euclidean distance, the regions associated to a pattern (called Voronoi regions) are delimited by linear borders. This can be modified if the distance is changed, or if a K-NN strategy is used, where the class assigned to a pattern is the most frequent class among its $\mathrm{K}$ nearest neighbors. K-NN strategy can approximate non-linear borders of the Voronoi regions. In this paper, however, we have consistently used the Euclidean distance to calculate distances between particles and prototypes.

Both computational reasons and presence of noise in data led to the development of techniques that reduce the number of patterns evaluated without increasing the classification error. These methods calculate a reduced set of prototypes, that are the only ones used for classification. In instance selection methods ${ }^{16)}$ prototypes are a subset of the pattern set, but this is not true for all the prototype methods ${ }^{17,18)}$. Among these methods we shall use LVQ for comparison.

In this paper, we use PSO to find a collection of prototypes that do not belong to the problem data, but represent the training pattern set and can classify the patterns according to the class of the nearest prototype in the collection.

In a standard approach to this problem using PSO, we would have decided the number of prototypes to use and we would have encoded the positions of all the prototypes and their classes in each particle. This approach has several 
disadvantages:

- Dimension of the search space grows with the number of prototypes; dimension is equal to the number of attributes times the number of prototypes in the solution.

- The maximum number of prototypes in the solution must be fixed at the start of the algorithm. It cannot grow if this number is not adequate to the problem.

In order to apply PSO to the problem avoiding the increase in the search space dimension, we want to test a different approach that we call the Michigan Approach. This term is borrowed from the area of genetic Classifier Systems 19, 20).

The Michigan approach was applied to PSO, using the binary version of PSO discover a set of induction rules for discrete classification problems ${ }^{11)}$. In that work the Michigan approach was able to reach better solutions in a much lower number of rule evaluations.

In this approach, each particle represents a single prototype. The solution is a collection of particles instead of a single particle. For this approach to work, the standard PSO has to be modified to ensure that the particles do not converge; instead the swarm evolves toward a configuration where each particle may be considered part of a collective solution. As the resulting algorithm is different from the standard PSO algorithm, some testing is required to perform selection of proper values for two of the algorithm parameters: $\chi$, that is a scale factor that influences the speed of the particle, and the number of particles used for the experiments.

The advantages of the Michigan approach versus the conventional PSO approach are:

- computational cost, as particles have much lower dimension

- flexibility and reduced assumptions, as the number of prototypes in the solution is not fixed.

This paper is organized as follows: Section 2 shows how the solution to the classification problem is encoded in the particles of the swarm; Section 3 details the modifications made to the original PSO algorithm to implement the Michigan approach and adapt it to classification problems; Section 4 describes the experimental setting and results of experimentation; finally, Section 5 discusses our conclusions and future work related to the present study. 


\section{$\S 2 \quad$ PSO Algorithm Encoding Prototypes}

In the Michigan-approach PSO we propose, each particle represents a potential prototype to be used to classify the patterns using the nearest neighbor rule. The particle position will be interpreted as the position of a prototype. Each particle has also a class; this class does not evolve following the PSO rules, but remains fixed for each particle since its creation.

For a given problem, a particle swarm is created with an equal share of particles of each of the classes present in the training set.

The following Table 1 represents the structure of a swarm with $N$ particles each of which corresponds to a single prototype, in a problem with $D$ attributes and $K$ classes. Among the whole population of the swarm, classes are assigned from 0 to $\mathrm{K}-1$ to the first $\mathrm{K}$ particles, and the sequence is repeated until prototype $\mathrm{N}\left(\right.$ so $\left.C_{i}=i \bmod K\right)$.

Table 1 Encoding of a set of prototypes in a whole swarm in the Michigan PSO. The swarm may encode $N$ prototypes in a problem of $D$ attributes

\begin{tabular}{|c|c|c|}
\hline & Position & Class \\
\hline Particle 1 & $X_{11} X_{12} \ldots X_{1 D}$ & $C_{1}$ \\
\hline Particle 2 & $X_{21} \quad X_{22} \ldots X_{2 D}$ & $C_{2}$ \\
\hline $\begin{array}{l}\ldots \\
\text { Particle N }\end{array}$ & $\begin{array}{cccc} & \cdots & \\
X_{N 1} & X_{N 2} & \ldots & X_{N D}\end{array}$ & $\stackrel{\cdots}{C_{N}}$ \\
\hline
\end{tabular}

The swarm runs until a stopping criterion is met, and then the resulting particles positions are interpreted as the positions of the prototypes of the nearest neighbor classifier.

\section{$\S 3 \quad$ MPSO Algorithm Modifications}

In the sections that follow, we introduce the pseudocode for the new algorithm (MPSO). Some concepts that differ over the standard PSO are introduced in the pseudocode and are explained in detail in the referenced subsections.

The basic variations in equations are the introduction of a repulsion force and the use of a dynamic definition of the neighborhood of a particle. When moving, each particle selects another one from what we call a "non-competing" set as a leader for attraction, and a second one from a "competing" set as a leader for repulsion. Both neighborhoods are defined dynamically on each iteration and take into account the particles' classes. In this definition, particles compete with particles of the same class but cooperate with particles of different classes. 
Also the concept of "local fitness" is used. A single particle is measured as "good" if it classifies correctly patterns in its surroundings (more precisely, in its Voronoi region). The formula for the particle fitness does not take into account how the rest of the patterns are classified.

\subsection{Modified PSO pseudo code and movement}

The factors above replace the standard PSO implementation. The overall procedure can be described as follows:

1. Load training patterns

2. Initialize swarm.

3. Insert $\mathrm{N}$ particles of each class in the training patterns.

4. Until max. number of iterations reached or success rate is $100 \%$ :

a. Calculate which particles are in the competing and non-competing sets of particles for every class (see Sect. 3.4).

b. For each particle,

1 Calculate Local Fitness (see Sect. 3.3).

2 Calculate Social Adaptability Factor (see Sect. 3.5).

3 Find the closest particle in the non-competing set for the particle class (attraction center).

4 Find the closest particle in the competing set for the particle class (repulsion center).

5 Calculate the particle's next position based on its previous velocity, previous best position, attraction center and repulsion center (see (3) in Sect. 3.2).

c. Move the particles

d. Assign classes to the patterns in the training set using the nearest particle.

e. Evaluate the swarm classification success

f. If the swarm gives the best success so far, record the current positions of the particles as "current best swarm".

5. Delete, from the best swarm found so far, the particles that can be removed without a reduction in the classification success value.

6. Evaluate the swarm classification success over the validation set and report result.

Note that a reduction algorithm is applied after the swarm reaches its maximum number of iterations. Starting with the particle with the worse local fitness value, particles are marked for removal one at a time; if this action does not reduce the swarm classification success over the training set then the particle is removed. This procedure is iterated for all the particles. The resulting swarm is considered the algorithm solution and is then evaluated using the validation set. 
In preliminary versions of the algorithm we performed particle deletion during the evolution, but that decreased its performance. The reason was that, during evolution, particles easily oscillate from good to very bad local fitness values. This made the cleaning algorithm remove too many particles and reduce the swarm size too much.

\subsection{Modified PSO Equation}

In order to take into account the modifications previously described, the equation that determines the velocity at each iteration in the basic PSO algorithm (1) has to be modified. In our work the velocity change is given by (3).

$$
\begin{aligned}
& v_{i d}^{t+1}=\chi\left(w \cdot v_{i d}^{t}+c_{1} \cdot \psi_{1} \cdot\left(p_{i d}^{t}-x_{i d}^{t}\right)+\right. \\
& c_{2} \cdot \psi_{2} \cdot \operatorname{sign}\left(a_{i d}^{t}-x_{i d}^{t}\right) \cdot S f_{i}+ \\
& \left.c_{3} \cdot \psi_{3} \cdot \operatorname{sign}\left(x_{i d}^{t}-r_{i d}^{t}\right) \cdot S f_{i}\right) .
\end{aligned}
$$

Where symbols that were already in 1 still have the same meanings, and the meanings of the new symbols are as follows:

$c_{3} \quad$ new constant repulsion weight factor

$a_{i} \quad$ attraction center for particle $i$

$r_{i} \quad$ repulsion center for particle $i$

$S f_{i} \quad$ Social Adaptability Factor, inversely dependent on the particle fitness; and $\psi_{3} \quad$ random factor in the $[0,1]$ interval

Note that the repulsion term is weighted by a random factor $\left(\psi_{3}\right)$ and a fixed weight $\left(c_{3}\right)$. This weight becomes a new parameter for the algorithm.

If either $a_{i}$ or $r_{i}$ does not exist, the respective term is ignored.

\subsection{Local Fitness Function}

In the Michigan approach, a local fitness value has to be calculated for each particle. This function is used to determine which is the best position found by the particle. As each particle represents a single prototype, the function takes into account the patterns to which the particle is the closest in the whole swarm. The area in which those patterns are located is the Voronoi region of the prototype represented by the particle. Those patterns are the ones that are "classified" by the particle. 
We also included an additional factor. We prefer particles that are close to the patterns they classify. For this purpose, we first calculate the $G_{f}$ and $B_{f}$ values using (4). The $G_{f}$ factor evaluates how many patterns the particle classifies correctly, and how close the particle is to those patterns. The $B_{f}$ factor is calculated using the patterns that the particle classifies incorrectly.

$$
\begin{aligned}
G_{f} & =\sum_{\{g\}} \frac{1}{d_{g, i}+1.0} \\
B_{f} & =\sum_{\{b\}} \frac{1}{d_{b, i}+1.0}
\end{aligned}
$$

Where $\{g\}$ is the set of patterns of the same class classified by the particle; $\{b\}$ is the set of patterns of different class classified by the particle; and $d_{g, i}, d_{b, i}$ are the Euclidean distances between the patterns and the particle.

Constant values 1.0 are just a way to ensure that no infinite values are assigned to $G_{f}$ and $B_{f}$. Particles may be initialized or placed "over" patterns if desired at any moment of the algorithm evolution.

Local Fitness is then calculated using the $G_{f}$ and $B_{f}$ factors in (5).

$$
\text { Local Fitness }= \begin{cases}0 & \text { if }\{g\}=\{b\}=\emptyset \\ \frac{G_{f}}{N_{\mathrm{P}}}+2.0 & \text { if }\{b\}=\emptyset \\ \frac{G_{f}-B_{f}}{G_{f}+B_{f}}+1.0 & \text { otherwise }\end{cases}
$$

Where $\{g\}$ and $\{b\}$ have the same meanings as in (4) and $N_{P}$ is the number of patterns in the training set.

In the fitness function, two constants (2.0 and 1.0) are used to define two distinct intervals for particles that have different characteristics in terms of patterns they classify:

- Particles that classify patterns both of their own class (true positives) and of different classes (false positives) have fitness values in the range $[0.0,2.0)$. In this range, the function only takes into account local information.

- Particles that only classify patterns of their own class have fitness value greater than 2.0, and so those positions are always preferred over the previous. In this range, local fitness uses some global information: the 
total number of patterns to be classified. In this way, particles with $\{b\}=\emptyset$ are still given different fitness values.

By using the distance between the pattern and the particle we give higher fitness to particles close to the patterns they classify, so they can move closer to the area where the patterns are located. This tendency may be compensated by the social terms.

\subsection{Neighborhood for the Michigan PSO}

For the success of the Michigan approach, the algorithm has to avoid convergence toward a single high-fitness particle, because this particle can only represent a very limited solution (only one prototype).

In the standard PSO, the attractive sociality term in (1) tries to move particles close to positions with good fitness. To change this behavior, in the Michigan approach we introduce several modifications that are based on using the particle class to define its neighborhood and divide the swarm into competing and non-competing particles:

- For each particle of class $C_{i}$, non-competing particles are all the particles of classes $C_{j} \neq C_{i}$ that classify at least one pattern of class $C_{i}$.

- For each particle of class $C_{i}$, competing particles are all the particles of class $C_{i}$ that classify at least one pattern of that class $\left(C_{i}\right)$.

When the movement for each particle is calculated, that particle is both:

1. Attracted by the closest (in terms of Euclidean distance) non-competing particle in the swarm, which becomes the "attraction center" for the movement. In this way, non-competing particles guide the search for the patterns of different class.

2. Repelled by the closest competing particle in the swarm, which becomes the "repulsion center" for the movement. In this way, competing particles retain diversity and push each other to find new patterns of their class in different areas of the search space.

The rules above achieve the following results:

- A particle is only attracted by those particles that misclassify patterns. In that case, it is only affected by the closest particle that meets that criterion. Hence, particles may cluster around different points of the search space instead of converging toward a single position.

- The way repulsion is defined means that, when several particles of a class 
are close to a cluster of patterns of that class, instead of converging toward the cluster center (or elsewhere the position that maximizes the fitness function), they can stay near the border of the cluster. This allows more accurate classification, as particles are able to locate the decision frontier where classification is harder.

Other authors have already used the idea of repulsion in PSO in different ways. For instance, Blackwell ${ }^{21,22)}$, introduces repulsion to increase population diversity in the standard PSO and allow the swarm to dynamically adapt to change.

\subsection{Social Adaptability Factor}

The social part of the algorithm (influence from neighbors) determines that particles are constantly moving toward their non-competing neighbor and far from their competing neighbor. However, particles that are already located in the proximity of a good position for a prototype should rather try to improve their position and should possibly avoid the influence of neighbors.

To implement this effect we have generalized the influence of fitness in the sociality terms by introducing a new term in the PSO equations, called "Social Adaptability Factor" $\left(S_{f}\right)$, that depends inversely on the "best fitness" of the particle. In particular, we have chosen plainly the expression in (6).

$$
S f_{i}=1 /(\text { Best Local Fitness } i+1.0) .
$$

This coefficient should be positive. This requirement has to be taken into account in the definition of the local fitness function. With the current Local Fitness function the value for $S_{f}$ is in the range $[0.25,1]$.

This factor contributes to the swarm stability because, as particles evolve toward better positions, they are less likely to move away from those positions, as the individual component of the particle velocity is not affected by this factor as shown in (3).

\section{$\S 4 \quad$ Experimentation}

In this section we describe the results of two sets of experiments. The first one is aimed to understand the influence of the algorithm's parameters in the experiment results, and the second one is performed in order to compare those results with other classification algorithms. 


\subsection{Global Swarm Evaluation}

The local fitness function, defined above, is used for the particles movement. However, to evaluate the goodness of the swarm as a classifier system, we use the classification success rate $(7)$.

$$
\text { Swarm Evaluation }=\frac{\text { Good classifications }}{\text { Total patterns }} \cdot 100
$$

Given the nearest neighbor criterion for classification, unclassified patterns are not possible, as every pattern is assigned the class of the nearest prototype.

The system stores the best swarm evaluation obtained when performing classification on the training set. This function is also used to evaluate the final best swarm success rate over the validation set.

\subsection{Problems' Description and Basic Experimentation Setup}

We perform experimentation on the problems summarized in Table 2. The first two (Clusters and Diagonal) are artificial problems and they are used to understand the new algorithm's properties; the rest are well-known real problems taken from the UCI collection, used for comparison with other classification algorithms.

Table 2 Problems used in the experiments

\begin{tabular}{lrcccl}
\hline Name & Instances & Attrbs. & Classes & Class Distribution & Validation \\
\hline Clusters & 80 & 2 & 2 & $40 / 40$ & Train set only \\
Diagonal & 2000 & 2 & 2 & $1000 / 1000$ & Train \& Test \\
Diabetes & 768 & 8 & 2 & $500 / 268$ & 10 -fold CV \\
Bupa & 345 & 6 & 2 & $200 / 145$ & 10 -fold CV \\
Glass & 214 & 9 & 6 & $70 / 76 / 17 / 13 / 9 / 29$ & 10 -fold CV \\
Thyroid (new) & 215 & 5 & 3 & $150 / 35 / 30$ & 10 -fold CV \\
Wisconsin & 699 & 6 & 2 & $458 / 241$ & 10 -fold CV \\
\hline
\end{tabular}

The Cluster problem is a very simple problem with five different clusters, randomly generated and clearly separated.This problem was only used to provide a clear graphic representation of the type of solutions found by the algorithm, and not for comparison.

The Diagonal problem is a bi-dimensional problem that is very simple for linear classifiers. We generate 500 random training patterns and 1500 validation 
patterns with coordinates in the $[0,1]$ range. Patterns where $x>y$ are assigned class 1 , and the rest are assigned class 0.

For UCI problems, attributes values were normalized to the $[0,1]$ interval. As prototypes are placed in the same search space as patterns, the same range was also used to constrain the particles' positions.

Velocity was clamped to the interval $[-1.0,+1.0]$ and $w=0.1$ in all the experiments.

Table 3 shows the parameters used for both the artificial and the UCI problems.

It is clear that problem-specific tuning may provide better results than selection of a single set of parameters for all the problems. However, we require some starting values which may later be adjusted for the different problems.

For the UCI problems, we tried to find a fixed proportion between $c_{1}$, $c_{2}$ and $c_{3}$ which gives acceptable results on a variety of problems. Our starting decision was to keep $c_{1}=c_{2}=1.0$ so only the value for $c_{3}$ had to be adjusted. The choice of $c_{1}=c_{2}$ is very common when constricted PSO is used ${ }^{23)}$. The initial value for $c_{3}$ was selected after some preliminary experimentation.

For the artificial problems, we used smaller values for the PSO parameters $\left(c_{1}, c_{2}\right.$, and $\left.c_{3}\right)$ which lead to slower evolution but more accurate results.

Table 3 Parameters used for each of the problems

\begin{tabular}{lcccrc}
\hline Problem & $\begin{array}{c}\text { Itera- } \\
\text { tions }\end{array}$ & $\mathrm{w}$ & $c_{1}$ & $c_{2}$ & $c_{3}$ \\
\hline Artificial Problems & 100 & 0.1 & 0.5 & 0.15 & 0.05 \\
UCI Problems & 300 & 0.1 & 1.0 & 1.0 & 0.25 \\
\hline
\end{tabular}

We performed 100 runs of the algorithm for the Cluster problem and Diagonal problems, and 10 runs of the problems that use 10-fold cross validation, which gives a total of 100 runs over each.

On each run, after the given number of iterations is reached, the best solution found for the training set is cleaned (useless particles are removed) and the resulting swarm is used to classify the validation set.

In the result tables, the success rate is averaged over the total number of runs; we also provide the best result over the set of experiments. Wherever cross-validation was used we provide the best of the 10 different runs.

Regarding the parameters, we experimentally found that a small value of $w$ lead to better results in all cases. The number of iterations (300) was selected 
after checking that number was roughly equal to double the average iteration in which the best result was achieved.

Values for the rest of the parameters were selected after performing some experimentation as shown in the following sections.

\subsection{Number of Particles}

In MPSO the number of particles in the swarm must be fixed at the start of the algorithm. We were interested in the importance of the number of particles on the algorithm result.

With this goal we ran a series of experiments with increasing values of the number of particles per class $(5,10,20$ and 40$)$. We used $\chi=1.0$ and the rest of the parameters were kept as in Table 3 .

Results in Table 4 show that the increase in the number of particles also increases accuracy. The effect depends on the problem, and is more important in the Diagonal, Bupa, Glass and Thyroid problems. The increment is gradual and we must note that even the experiment with 5 particles per class has good results in all the problems.

Table 4 Best swarm success rate (in \%) with different values of the number of particles per class

\begin{tabular}{crrrrrr}
\hline $\begin{array}{c}\text { Particles } \\
\text { per Class }\end{array}$ & Diagonal & Diabetes & Bupa & Glass & Thyroid & Wisconsin \\
\hline 5 & 94.60 & 73.45 & 62.89 & 81.61 & 93.33 & 95.80 \\
10 & 96.14 & 74.14 & 63.29 & 82.04 & 94.03 & 96.46 \\
20 & 96.88 & 74.68 & 64.28 & 83.46 & 93.63 & 96.37 \\
40 & 97.31 & 74.67 & 65.63 & 85.05 & 95.31 & 96.80 \\
\hline
\end{tabular}

However this improvement must be balanced with the increase in the number of prototypes in the solution shown in Table 5. Obviously a greater number of particles in the swarm requires higher computing costs.

As the balance between accuracy and computational cost is important, this experiment points toward the implementation of some automatic (adaptive) method of increasing or decreasing population as needed during the run of the algorithm.

\subsection{Experimental Selection of the $\chi$ Coefficient}

The proposed algorithm has two weight parameters originating from the 
Table 5 Average number of prototypes in the solution, with different values of the number of particles per class

\begin{tabular}{crrrrrr}
\hline $\begin{array}{c}\text { Particles } \\
\text { per Class }\end{array}$ & Diagonal & Diabetes & Bupa & Glass & Thyroid & Wisconsin \\
\hline 5 & & & & & & \\
10 & 17.55 & 6.39 & 6.48 & 8.07 & 7.74 & 6.03 \\
20 & 33.45 & 10.47 & 10.89 & 10.30 & 11.37 & 11.12 \\
40 & 57.67 & 29.50 & 29.08 & 17.99 & 22.08 & 40.63 \\
\hline
\end{tabular}

standard PSO model $\left(c_{1}\right.$ and $\left.c_{2}\right)$ and includes the $c_{3}$ weight for the repulsion term.

To simplify experimentation, we decided to start by keeping a fixed proportion between them and only vary the constriction factor, $\chi$. Lower values for this parameter mean that the increments in velocity are smaller, so search is performed in smaller steps.

For these experiments, we used 10 particles per class. Results are shown in Table 6. For the Diagonal, Bupa, Glass and Thyroid problems results are better when a small value of $\chi$ is used. Differences are very small in the rest of the problems. Also, Table 7 shows that smaller values of $\chi$ allow more prototypes in the solution.

Table 6 Best swarm success rate (in \%) with different values of the $\chi$ coefficient

\begin{tabular}{rrrrrrr}
\hline$\chi$ & Diagonal & Diabetes & Bupa & Glass & Thyroid & Wisconsin \\
\hline 0.25 & 96.20 & 73.76 & 64.42 & 87.64 & 95.68 & 96.24 \\
0.50 & 96.14 & 74.25 & 64.76 & 86.27 & 95.90 & 96.50 \\
0.75 & 95.85 & 73.88 & 64.51 & 84.60 & 94.61 & 96.43 \\
1.00 & 96.14 & 74.14 & 63.29 & 82.04 & 94.03 & 96.46 \\
\hline
\end{tabular}

Comparison with Table 4 and Table 5 suggest show that for the Glass and Thyroid problems we can obtain similar solutions in terms of quality and number of prototypes with half the number of particles if we reduce the value of $\chi$ to 0.25 or 0.5 , with a reduced computing cost.

From these results we recommend a value of $\chi=0.5$, which means that actual values of the $c_{i}$ weights are half of the values initially set. It must be noted that performance may be improved for specific problems by tuning the $c_{i}$ coefficients independently in a neighborhood of the values used in this study. 
Table 7 Average number of prototypes in the solution, with different values of the $\chi$ coefficient

\begin{tabular}{rrrrrrr}
\hline$\chi$ & Diagonal & Diabetes & Bupa & Glass & Thyroid & Wisconsin \\
\hline 0.25 & 15.41 & 11.53 & 12.78 & 18.81 & 16.30 & 12.28 \\
0.50 & 15.04 & 11.28 & 11.75 & 15.58 & 16.33 & 11.99 \\
0.75 & 14.69 & 11.15 & 11.47 & 12.46 & 13.53 & 11.53 \\
1.00 & 17.39 & 10.47 & 10.89 & 10.30 & 11.37 & 11.12 \\
\hline
\end{tabular}

\subsection{Discussion of the Results}

In this section we will discuss some of the results of our experimentation. We have chosen the results of the series of experiments that use 10 particles per class and $\chi=0.50$ for discussion as they provide good results for all the problems with a small number of prototypes in the solution.

The two artificial experiments are used to show the behavior of the swarm with the proposed modifications. This can be easily shown plotting some of the solutions found by the swarm for these problems.

In Fig. 1 we represent one of the solutions for the "Cluster" problem. It shows how the attraction and repulsion rules make the particles group around the different clusters but also how the particles of the same class remain separated (due to the repulsion force). Once the swarm reaches this kind of configuration, attraction forces are null (no particle classifies patterns of the wrong class), and repulsion forces are compensated if they move the particles toward positions of lower local fitness, so the particles remain almost stable in positions near the optimum. In this problem, the swarm easily finds and separates the clusters every time.

In Table 8 we summarize the results for all the problems. The table shows the best swarm success rate (in \%) on the validation and training set, averaged over all the runs of the experiment and all the folds when 10-fold validation is used; "Evaluations" is calculated as the average number of iterations needed to find the best result for each individual run, times the number of particles in the swarm; "Best Experiment" is the best success rate among all the runs of each experiment; and "Prototypes" is the average number of prototypes kept after cleaning the best swarm.

\subsection{Comparison with other Classification Algorithms}

In this section we compare the results of MPSO on the UCI data sets 


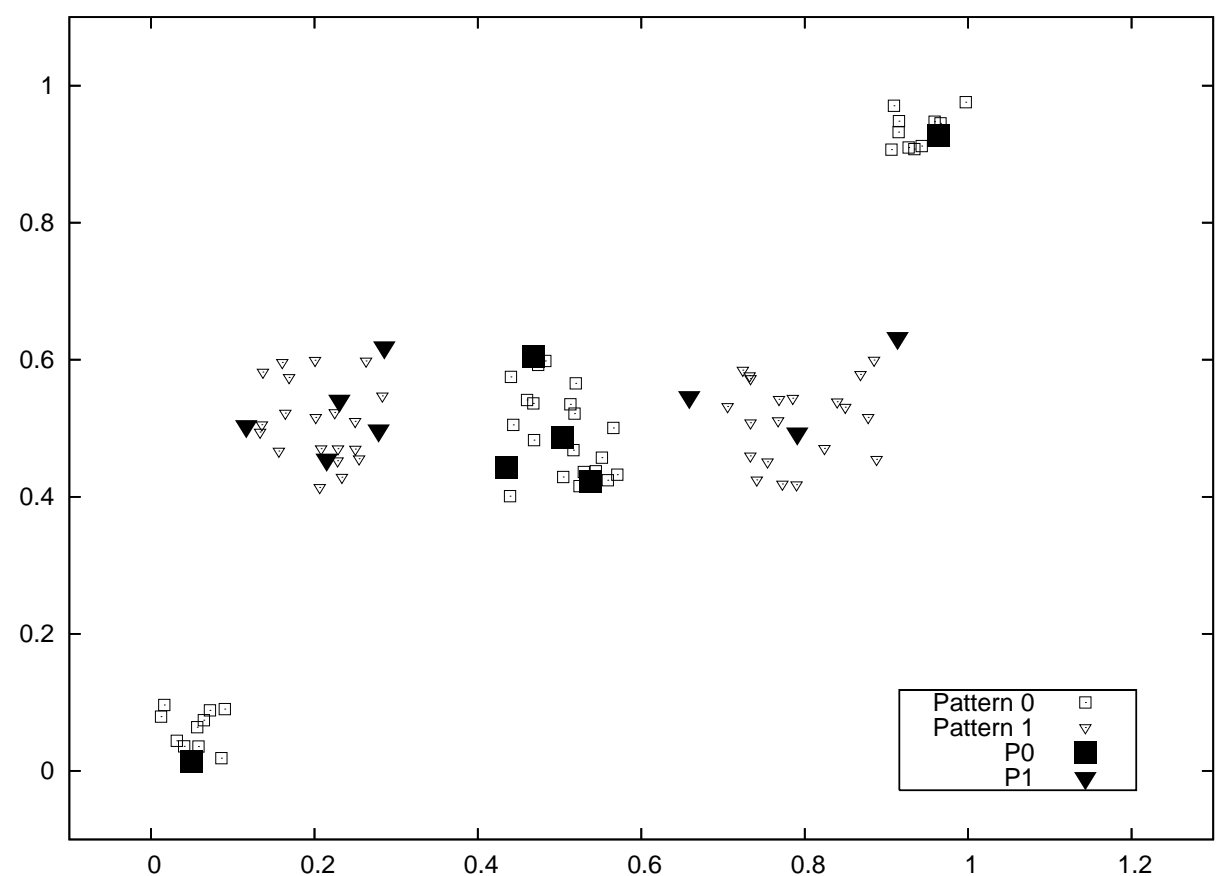

Fig. 1 Sample solution for the problems "Cluster".Patterns are outlined, particles are solid. Particles locate clusters and cluster boundaries.

with the results of other algorithms. As our algorithm performs classification using the NN rule, we first compare MPSO with related methods, such as the basic 1-NN algorithm, 3-NN algorithm and LVQ (Learning Vector Quantization) method, that is indeed a prototype selection algorithm. All those methods may share common limitations, as all of them are based on the same distance measure on the search space. Then, we perform the same comparison with algorithms that are based on completely different strategies.

In all comparisons, two-tailed t-tests with significance level of 0.05 were performed in order to determine which are the best algorithms for these problems. The plus and minus signs determine the result of the algorithm versus MPSO; that is, a " +" sign means that the algorithm performed significantly better than MPSO, and a "-" sign means that the algorithm performed significantly worse than MPSO.

In Table 9 we compare our results on the UCI data sets with the results of our own tests with other Nearest Neighbor algorithms, performed using 
Table 8 Experiment results for the PSO with Michigan approach (MPSO)

\begin{tabular}{lrrrrr}
\hline Problem & $\begin{array}{r}\text { Training } \\
\text { Set }\end{array}$ & $\begin{array}{r}\text { Validation } \\
\text { Set }\end{array}$ & Evaluations & Prototypes & $\begin{array}{r}\text { Best } \\
\text { Experiment }\end{array}$ \\
\hline Cluster & 100 & N/A & 378 & 12.21 & 100 \\
Diagonal & 97.24 & $96.14 \pm 1.21$ & 3453 & 15.04 & 96.73 \\
\hline Bupa & 66.15 & $64.76 \pm 2.31$ & 2827 & 11.75 & 67.82 \\
Diabetes & 74.44 & $74.25 \pm 1.18$ & 3304 & 11.28 & 76.62 \\
Glass & 87.83 & $86.27 \pm 2.29$ & 8596 & 15.58 & 90.11 \\
Thyroid & 96.42 & $95.90 \pm 0.88$ & 4955 & 16.33 & 97.10 \\
Wisconsin & 96.50 & $96.50 \pm 0.26$ & 3576 & 11.99 & 96.94 \\
\hline
\end{tabular}

WEKA ${ }^{24)}$ toolkit. In the table, $\operatorname{IBK}(\mathrm{k}=1)$ means plain $\mathrm{NN}, \operatorname{IBK}(\mathrm{k}=3)$ is 3 NN classification and LVQ is Learning Vector Quantization ${ }^{18)}$, which is also a prototype method.

The success rate in terms of accuracy for MPSO is comparable or clearly better than the other algorithms in all the UCI problems except the Thyroid problem.

In this latter case our hypothesis is that more particles are needed to achieve the performance of 1-NN classification (note that in this case 1-NN is the winning algorithm). This fact suggests that an adaptive version of MPSO that included reproduction and/or deletion of particles might be useful to reduce the importance of a correct setting of the initial number of particles. This hypothesis seems to be backed up by the increase in accuracy shown when the number of particles grows in Table 4.

Concerning the Diabetes problem, MPSO significantly improves the basic 1-NN algorithm but is only equal to $3-\mathrm{NN}$ and LVQ. In this problem it is known that both higher $\mathrm{K}$ values in $\mathrm{K}-\mathrm{NN}$ classification and non-Euclidean distances achieve better results. The best result found in literature using nearest neighbor classifiers on the Diabetes problem was obtained with $\mathrm{K}=23$ and Manhattan distance ${ }^{25)}(76.7 \pm 4.0 \%)$ so our hypothesis is that usage of a nonEuclidean distance is required to be able to improve the results in this problem.

In the Glass problem our algorithm significantly improves the results of all the other classifiers. This is to our knowledge the best result over this data set. Some values from other algorithms for the Glass problem can be found in literature but all are far from the accuracy of MPSO (C.50 66.3\% , 5-NN 67.62\%, SMO $62.86 \%{ }^{26)}$. Even with 5 particles per class, MPSO clearly gives a very good result (81.61\%, see Sect. 4.3) so MPSO seems to be very well-suited to this 
Table 9 Success rate on validation data: average results and standard deviations

\begin{tabular}{lrrrr}
\hline & MPSO & IBK 1 & IBK & LVQ \\
Problem & & $(\mathrm{K}=1)$ & \multicolumn{1}{c}{$(\mathrm{K}=3)$} \\
\hline Bupa & $\mathbf{6 4 . 7 6} \pm 2.31$ & $62.22 \pm 1.15(-)$ & $62.48 \pm 1.48(-)$ & $62.18 \pm 2.13(-)$ \\
Diabetes & $\mathbf{7 4 . 2 5} \pm 1.18$ & $70.40 \pm 0.56(-)$ & $\mathbf{7 3 . 8 9} \pm 0.56(=)$ & $\mathbf{7 4 . 2 6} \pm 0.86(=)$ \\
Glass & $\mathbf{8 6 . 2 7} \pm 2.29$ & $69.07 \pm 1.47(-)$ & $69.05 \pm 1.13(-)$ & $62.56 \pm 1.74(-)$ \\
Thyroid & $95.90 \pm 0.88$ & $\mathbf{9 6 . 9 3} \pm 0.57(+)$ & $94.31 \pm 0.70(-)$ & $91.03 \pm 1.35(-)$ \\
Wisconsin & $\mathbf{9 6 . 5 0} \pm 0.26$ & $95.37 \pm 0.17(-)$ & $\mathbf{9 6 . 5 4} \pm 0.30(=)$ & $95.88 \pm 0.28(-)$ \\
\hline
\end{tabular}

particular problem.

\section{$\S 5$ Conclusions}

The purpose of this paper is to study the performance of PSO in classification problems with continuous attributes. We will use PSO to determine a set of prototypes that represent the training patterns and that it can be used as a classifier using the nearest neighbor (1-NN) rule.

This task might be performed using a straightforward PSO algorithm by encoding a series of prototypes in each particle. However, this approach would have the disadvantage of producing a search space of high dimension that would lead to poor performance and high computational costs.

For this reason, we propose a Michigan Approach PSO (MPSO), in which each particle represents a single prototype (not a set of prototypes). In MPSO, each particle searches for a position that optimizes a Local Fitness function that only takes into account patterns in the particle's Voronoi region. The swarm movement equations and neighborhood functions are modified to ensure this behavior.

First, we performed experimentation on simple artificial problems to validate the swarm behavior and to determine the correct rules to be used. We also performed some experimentation in order to evaluate the influence in the performance of the algorithm of the number of particles in the swarm and the value of the $\chi$ parameter. The number of particles is important, as complex solutions require more prototypes; however, for the datasets we used, we obtained that 10 prototypes per class was enough. The $\chi$ parameter determines the size of the adjustment in velocity in each iteration, and may be decreased if needed. This increases the exploitation ability of the algorithm, and thus sometimes obtains a better performance, at the cost of a longer execution time.

Next, we tested the resulting swarm in five well-known benchmark prob- 
lems with parameter values obtained in the preliminary experimentation.

Results on the benchmark problems indicate that MPSO matches or outperforms the 1-NN classifier on most problems. This proves that MPSO can be used to produce a representative set of prototypes.

When the results are compared with algorithms of the same family (nearest neighbor and LVQ), MPSO produces competitive results in all the domains.

Given that we have not yet introduced k-NN classification (with $k>1$ ), attribute processing, nor any hybridization, we think these results are quite promising. However either attribute weighting or non Euclidean distances are likely to be required to compete in other problems.

Closer observation of the MPSO behavior also shows that this approach obtains a swarm with some characteristics which may be useful in other fields of application:

- Particles in the swarm are able to locate different areas with high values of the local fitness function and perform local search in those areas. This can be seen as multimodal optimization of the local fitness function, and can be of interest in that field. This behavior can also be useful in applications in image processing.

- Particles are able to find equilibrium situations which can be altered by the influence of intruders, forcing them to adapt to the new situation. This behavior can be of interest for dynamically-adapting swarms.

It is also interesting to address the issue of the number of particles in the swarm. MPSO can be improved by including a method for dynamic reproduction and deletion of particles. This would allow adaptive adjustment of this parameter to the requirements of the problem. However, before adding one of these methods to MPSO, some issues must be solved. First, any such mechanism will certainly add new parameters that should be given values either in an adaptive or in a problem-dependent way. In that case, further study would be required to give proper values or selection guidelines for those parameters. Second, population explosion should be prevented when it does not lead to performance improvement; this can be easy to do but may increase the computational cost of the algorithm. Third, stagnation can happen if particles are deleted too early.

Acknowledgment This article has been financed by the Spanish founded research project MSTAR::UC3M, Ref: TIN2008-06491-C04-03. 


\section{References}

1) J. Kennedy, R.C. Eberhart, and Y. Shi. Swarm intelligence. Morgan Kaufmann Publishers, San Francisco, 2001.

2) P. N. Suganthan. "Particle swarm optimiser with neighbourhood operator," In Proceedings of the IEEE Congress on Evolutionary Computation (CEC), pages 1958-1962, 1999.

3) R. Brits. Niching strategies for particle swarm optimization. Master's thesis, University of Pretoria, Pretoria, 2002.

4) X. Hu and R.C. Eberhart. "Multiobjective optimization using dynamic neighborhood particle swarm optimisation," In Proceedings of the IEEE Congress on Evolutionary Computation (CEC), pages 1677-16, 2002.

5) M. Clerc and J. Kennedy. "The particle swarm - explosion, stability, and convergence in a multidimensional complex space," IEEE Trans. Evolutionary Computation, 6(1):58-73, 2002.

6) F. van den Bergh. An analysis of particle swarm optimizers. PhD thesis, University of Pretoria, South Africa, 2002.

7) I. C. Trelea. "The particle swarm optimization algorithm: convergence analysis and parameter selection," Inf. Process. Lett., 85(6):317-325, 2003.

8) Riccardo Poli. "Dynamics and stability of the sampling distribution of particle swarm optimisers via moment analysis," Journal of Artificial Evolution and Applications, 2008:1-10, 2008.

9) T. Sousa, A. Silva, and A. Neves. "Particle swarm based data mining algorithms for classification tasks," Parallel Comput., 30(5-6):767-783, 2004.

10) A. Cervantes, P. Isasi, and I. Galván. "Binary Particle Swarm Optimization in classification," Neural Network World, 15(3):229-241, 2005.

11) A. Cervantes, P. Isasi, and I. Galván. "A comparison between the Pittsburgh and Michigan approaches for the Binary PSO algorithm," In Proceedings of the IEEE Congress on Evolutionary Computation 2005 (CEC 2005), pages 290-297, 2005.

12) Z. Wang, X. Sun, and D. Zhang. "Classification Rule Mining Based on Particle Swarm Optimization," In Rough Sets and Knowledge Technology, volume 4062/2006 of Lecture Notes in Computer Science, pages 436-441, Springer Berlin / Heidelberg, 2006.

13) Z. Wang, X. Sun, and D. Zhan. "A PSO-Based Classification Rule Mining Algorithm," In Advanced Intelligent Computing Theories and Applications. With Aspects of Artificial Intelligence, volume 4682/2007 of Lecture Notes in Computer Science, pages 377-384, Springer Berlin / Heidelberg, 2007.

14) Ahmed Ali Abdalla Esmin. "Generating fuzzy rules from examples using the particle swarm optimization algorithm," In Proceedings of the 7th International Conference on Hybrid Intelligent Systems (HIS 2007), pages 340-343. IEEE Computer Society, 2007.

15) I. De Falco, A. Della Cioppa, and E. Tarantino. "Evaluation of Particle Swarm Optimization Effectiveness in Classification," In Fuzzy Logic and Applica- 
tions, volume 3849/2006 of Lecture Notes in Computer Science, pages 164-171. Springer Berlin / Heidelberg, 2006.

16) H. Brighton and C. Mellish. "Advances in instance selection for instance-based learning algorithms," Data mining and knowledge discovery, 6(2):153-172, 2002.

17) F. Fernández and P. Isasi. "Evolutionary design of nearest prototype classifiers," Journal of Heuristics, 10(4):431-454, 2004.

18) T. Kohonen. Self-Organizing Maps. Springer Verlag, Berlin, 1995.

19) J.H. Holland. "Adaptation," In R. Rosen \& F. M. Snell (Eds.), Progress in theoretical biology, 4. New York: Plenum,pages 263-293. 1976.

20) S. W. Wilson. "Classifier fitness based on accuracy," Evolutionary Computation, 3(2):149-175, 1995.

21) T. M. Blackwell and P. J. Bentley. "Don't push me! collision-avoiding swarms," In Proceedings of the IEEE Congress on Evolutionary Computation (CEC), pages 1691-1696, 2002.

22) T. M. Blackwell and P. J. Bentley. "Dynamic search with charged swarms," In Proceedings of the Genetic and Evolutionary Computation Conference 2002 (GECCO), pages 19-26, 2002.

23) D. Bratton and J. Kennedy. "Defining a standard for particle swarm optimization," Swarm Intelligence Symposium, 200\%. SIS 200\%. IEEE, pages 120-127, 1-5 April 2007.

24) I. H. Witten and E. Frank. Data Mining: Practical machine learning tools and techniques. Morgan Kaufmann, San Francisco, 2005.

25) W. Duch. Datasets used for classification: comparison of results. http://www.phys.uni.torun.pl/kmk/projects/datasets.html.

26) G. Guo and D. Neagu. "Similarity-based classifier combination for decision making," Systems, Man and Cybernetics, 2005 IEEE International Conference on, 1:176-181 Vol. 1, Oct. 2005. 